\title{
Hazardous factors besides infection in hypoglycemia
}

\author{
YU-JANG SU ${ }^{1-4}$, YEN-CHUN LAI ${ }^{5}$ and CHIA-JUNG LIAO ${ }^{1}$ \\ ${ }^{1}$ Department of Emergency Medicine, Mackay Memorial Hospital, Taipei 10449; \\ ${ }^{2}$ Department of Oral Hygiene, College of Oral Medicine, Taipei Medical University, Taipei 110; \\ ${ }^{3}$ Department of Medicine, Mackay Medical College, New Taipei City 252; ${ }^{4}$ Mackay Junior College of Medicine, Nursing, \\ and Management, Taipei 11260; ${ }^{5}$ Department of Anesthesiology, Taiwan Adventist Hospital, Taipei 105, Taiwan
}

Received November 11, 2016; Accepted February 23, 2017

DOI: $10.3892 /$ br. 2017.872

\begin{abstract}
Hypoglycemia is one of the most common issues encountered in daily emergency practice. In addition to the treatment of hypoglycemia, certain other situations concomitant with hypoglycemia require further treatment. The aim of the present study was to compare demographic and clinical characteristics of infected [urinary tract infection (UTI), pneumonia or biliary tract infection (BTI)] vs. non-infected hypoglycemic patients to establish which hypoglycemic patients required further observation or hospitalization. This was a retrospective cross-sectional study of hypoglycemic ( $<60 \mathrm{mg} / \mathrm{dl}$ ) hospitalized patients. The patients were divided into two groups: Those with hypoglycemia and concurrent infection (UTIs, pneumonia or BTIs; $n=103$ ) and non-infected hypoglycemic patients $(n=83)$. Student's t-test was performed to analyze continuous data and the $\chi^{2}$ test was used to analyze categorical data. Infections included 62 UTI, 43 pneumonia and 5 BTI. Comparing between the infected and non-infected groups, no significant differences were identified between mean glucose or body temperature, concurrent diagnosis (liver cirrhosis, uremia, acute renal failure, stroke or cancer) or mortality rate. Compared with the non-infected subjects, the infected patients were significantly older and had a significantly higher female ratio, as well as increased heart rates, white blood cell count and C-reactive protein levels. The present study proposes that characteristics, including concurrent stroke, liver cirrhosis and cancer are a point of focus in the initial management of hospitalized hypoglycemic patients.
\end{abstract}

\section{Introduction}

Hypoglycemia is an endocrine emergency that induces an altered mental state in patients, resulting in lethargy, delirium,

Correspondence to: Dr Yu-Jang Su, Department of Emergency Medicine, Mackay Memorial Hospital, 92 Sec 2, Chung-Shan N Road, Taipei 10449, Taiwan

E-mail: yjsu.5885@mmh.org.tw

Key words: hypoglycemia, liver cirrhosis, acute renal failure, infection, mortality rate, elderly confusion and organ dysfunction. Common causes are concurrent infection, a lack of adequate food intake, chronic alcohol abuse, interactions among medications (as a result of polypharmacy), exhaustion from physical exertion and overdose of medications (insulin/oral hypoglycemic agent). In the past, there have been numerous articles describing the etiologies of hypoglycemia, including old age, lack of recent food intake, infection, chronic renal insufficiency, liver diseases and recurrent hypoglycemic episodes (1-3). Hypoglycemia was investigated in the present study via a retrospective review of records from December 2009 to February 2012 at a tertiary teaching medical center in northern Taiwan. Comparisons were made between hypoglycemic patients with and those without concurrent infection in terms of multi-variable parameters and outcomes.

\section{Materials and methods}

Data collection. Patient age, gender, heart rate, systolic blood pressure (BP) and diastolic BP were recorded at triage at the Mackay Memorial Hospital (Taipei, Taiwan; between December 2009 and February 2012), and a retrospective chart review of hospitalized hypoglycemic cases (serum glucose $<60 \mathrm{mg} / \mathrm{dl}$ ) was conducted to gather data regarding white blood cell count (WBC), serum glucose, C-reactive protein (CRP), glutamic oxaloacetic transaminase (GOT), creatinine, sodium, potassium, past history of liver cirrhosis, uremia, concurrent infection [urinary tract infection (UTI), pneumonia or biliary tract infection (BTI)], concurrent cancer/malignancy, length of stay, lack of recent food intake, status of acute renal failure (ARF) and concurrent stroke. Only hypoglycemic cases that had been hospitalized were enrolled in the current study; those that were discharged from the emergency department (ED) following treatment were excluded. In Taiwan, the majority of hypoglycemic cases are discharged from the ED following successful resuscitation and treatment. Only a small fraction of hypoglycemic patients were admitted to endocrinology wards due to overcrowding as a result of the national health insurance system.

Patients. One hundred and eighty-six patients (aged 26-98 years; mean \pm standard deviation, $70.5 \pm 15.3$ years) were enrolled in the current study, with patients divided into concurrent infection $(n=103)$ and non-infected $(n=83)$ 


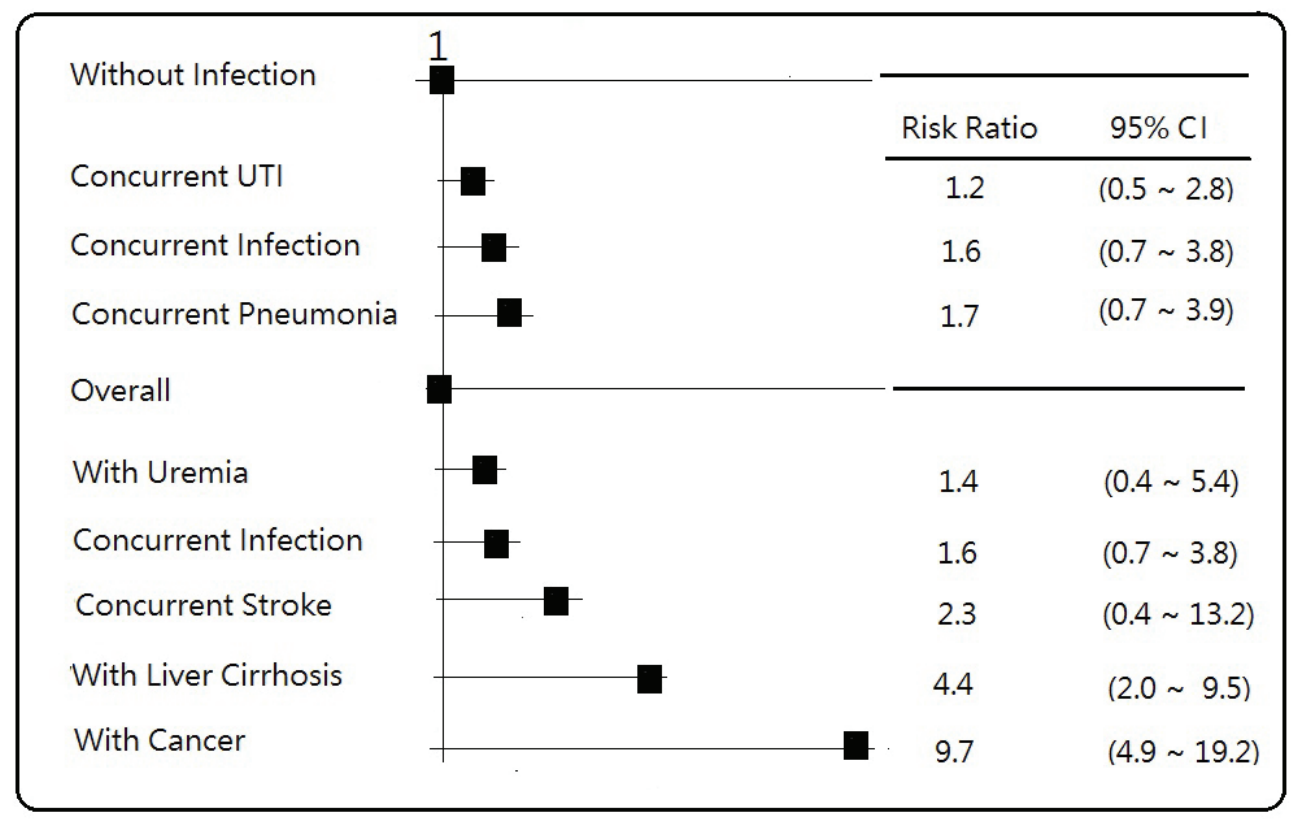

Figure 1. Risk ratio in mortality of hypoglycemic patients with concurrent infection and medical co-morbidities (uremia, stroke, liver cirrhosis and cancer). CI, confidence interval; UTI, urinary tract infection.

groups to compare the differences affecting the prognosis of hypoglycemia. Patients with concurrent infections were defined as hypoglycemic patients who were diagnosed with an infection during hospitalization, commonly including UTIs, pneumonia and BTIs. The current study was approved by the Institutional Review Board of Mackay Memorial Hospital (Taipei, Taiwan; approval no. 16MMHIS057e).

Patients with serum glucose levels $<60 \mathrm{mg} / \mathrm{dl}$ were defined as hypoglycemic (according to https://uihc. org/health-library/hypoglycemia-low-blood-sugar). ARF was defined as a doubling of the level of creatinine (4). Neonatal and pediatric patients were excluded. Instances of mortality were defined as those cases in which patients had succumbed prior to discharge. The basic data are presented in Tables I and II. There were 28 cases of hypoglycemia without a history of diabetes; of these, 4 cases were advanced hepatoma, 3 cases were alcoholism, 2 cases were esophageal cancer, 2 cases were upper gastrointestinal bleeding, 2 cases were asthma and chronic obstructive pulmonary disease, 2 cases were coronary artery disease and acute coronary syndrome, 2 cases were sepsis and multiple organ failure. In addition, there was 1 case of each of lung malignancy anorexia nervosa, gastric malignancy, hypoxic encephalopathy, subarachnoid hemorrhage (SAH) with five fractured ribs, history of cerebrovascular disease and bed ridden, cholangiocarcinoma, dilated cardiomyopathy, out-of-hospital cardiac arrest, peripheral arterial occlusive disease and 1 case of unknown etiology.

Statistical analysis. Multivariate analysis of BTI, UTI and pneumonia in the hypoglycemic cases was performed in the current study using commercial statistical software (SPSS version 11.0; SPSS, Inc., Chicago, IL, USA). Student's t-test, $\chi^{2}$ test and Fisher's exact test were performed and $\mathrm{P}<0.05$ was considered to indicate a statistically significant difference.

\section{Results}

One hundred and eighty-six hypoglycemia cases were examined. Of them, 28 hypoglycemic cases did not have a history of diabetes. Among these patients, $55.4 \%$ were infected during hospitalization (103/186), and of these, 60\% (62/103) had UTIs, $42 \%$ (43/103) suffered from pneumonia and $5 \%$ had BTIs. High CRP levels were noted in $82.8 \%$ (154/186) of the cases, $62.4 \%$ (116/186) had impaired renal function and $38.2 \%$ (71/186) exhibited elevated GOT. ARF accounted for $26.3 \%$ (49/186) of the hypoglycemic episodes.

Hypoglycemia with concurrent infection was identified to be more prevalent in elderly $(72.7$ vs. 67.7 ; $\mathrm{P}<0.05)$ and tachycardiac (89.2 vs. 83.1; $\mathrm{P}<0.05)$ patients, patients with elevated WBC counts $(12,430$ vs. 9,725; $\mathrm{P}<0.05)$, and patients with increased CRP levels (6.6 vs. $2.1 ; \mathrm{P}<0.01)$. In terms of gender, infection-associated hypoglycemia is female predominant (male:female ratio: 0.8 vs. $1.5 ; \mathrm{P}<0.05$ ). In terms of mortality rate, hypoglycemic patients with an infection were associated with a higher mortality rate than those without infection (13.6 vs. 8.4\%); however, this difference was not statistically significant $(\mathrm{P}=0.27)$. Comparisons between subjects with concurrent UTI and those without UTI, and comparisons between patients with concurrent pneumonia and those without pneumonia are presented in Table III. The risk ratios of concurrent infection and medical diseases were calculated and are presented in Fig. 1. The risk ratio of mortality in hypoglycemic patients was ranked from high to low as follows: with cancer (9.7), with liver cirrhosis (4.4), concurrent stroke (2.3), concurrent pneumonia (1.7), concurrent infection (1.6) and concurrent UTI (1.2).

\section{Discussion}

Hypoglycemia is a common emergency in daily practice. A previous animal study concluded that sepsis increases glucose 
Table I. Basic data of 186 hypoglycemic patients.

\begin{tabular}{lccc}
\hline Variable & Minimum & Maximum & Mean (standard deviation) \\
\hline Age (years) & 26 & 98 & $70.5(15.3)$ \\
Body temperature $\left({ }^{\circ} \mathrm{C}\right)$ & 31.8 & 39.1 & $36.4(1.2)$ \\
Heart rate $(\mathrm{bpm})$ & 0 & 139 & $86.5(18.1)$ \\
Systolic blood pressure $(\mathrm{mmHg})$ & 0 & 207 & $139.3(29.9)$ \\
Diastolic blood pressure $(\mathrm{mmHg})$ & 0 & 133 & $73.3(18.1)$ \\
White blood cell count $(/ \mu \mathrm{l})$ & 72 & 55000 & $11223.0(7198.1)$ \\
Glucose level (mg/dl) & 5 & 59 & $34.9(12.4)$ \\
C-reactive protein $(\mathrm{mg} / \mathrm{dl})$ & 0.04 & 42.35 & $5.1(6.5)$ \\
Glutamic oxaloacetic transaminase $(\mathrm{U} / \mathrm{l})$ & 12 & 3409 & $110.1(340.0)$ \\
Creatinine (mg/dl) & 0.2 & 13.9 & $2.8(2.6)$ \\
Sodium (meq/l) & 113 & 161 & $134.5(11.1)$ \\
Potassium (meq/l) & 2.0 & 7.6 & $4.1(1.0)$ \\
Hospital stay (days) & 1 & 161 & $15.2(18.9)$
\end{tabular}

Table II. Concurrent disorders of hypoglycemic patients.

\begin{tabular}{lrr}
\hline & \multicolumn{2}{c}{ Patients $(\mathrm{n}=186)$} \\
\cline { 2 - 3 } Disorder & $\mathrm{n}$ & $\%$ \\
\hline Type 2 diabetes mellitus & 155 & 83.3 \\
Without diabetes mellitus & 28 & 15.0 \\
Type 1 diabetes mellitus & 3 & 1.7 \\
Oral hypoglycemic agent control & 154 & 82.8 \\
Insulin control & 4 & 2.1 \\
Liver cirrhosis & 19 & 10.2 \\
Uremia & 13 & 7.0 \\
Concurrent infection & 103 & 55.4 \\
Urinary tract infection & 62 & 33.3 \\
Pneumonia & 43 & 23.1 \\
Biliary tract infection & 5 & 2.7 \\
Inadequate food intake & 83 & 44.6 \\
Acute renal failure & 49 & 26.3 \\
Concurrent cancer & 16 & 8.6 \\
Concurrent stroke & 4 & 2.2 \\
Mortality rate & 21 & 11.3 \\
\hline
\end{tabular}

uptake in the liver, spleen, lungs, ileum and skin; therefore, hypoglycemic patients are usually examined for potential concurrent infections (5). Of all concurrent infections, UTIs $(33.3 \%)$ were more commonly observed than pneumonia (23.1\%) and BTIs (2.7\%). Clinically, hypoglycemia itself results in variable symptoms of cranial dysfunction and altered mental conditions; in addition, infection and sepsis demonstrate these symptoms. The etiologies of hypoglycemia result from missed meals, infectious diseases, tumor, stroke, glyburide use, polypharmacy, renal insufficiency, loss of diabetic care or iatrogenic reasons (6).

Hypoglycemia occurs most frequently in the elderly, with $>70 \%$ of the hypoglycemic cases examined in the present study aged $>65$ years. In a Korean report conducted in 2012, the mean age of hypoglycemic patients was 69.5 years, which is comparable with the current study (age, 70.5 years) (1). In the present study, the mean age of the group of hypoglycemic patients with concurrent infection was 72.7 years, which was five years older than patients without infection $(7,8)$.

Hypoglycemia with concurrent infection is female predominant with a male:female ratio of 0.8 vs. 1.5 for non-infected patients. The reason for this ratio may have been due to the fact that, of the 62 hypoglycemic patients with concurrent UTIs in the present study, 43 were female $(69.4 \%)$. Thus, the ratio of males to females was a limitation of the current study.

Hypoglycemic patients with concurrent infections exhibited faster heartbeats (six more beats per minute) and higher CRP levels (6.6 vs. 2.1; $\mathrm{P}<0.01)$ when compared with the non-infected patients, and obvious leukocytosis owing to concurrent infection. In 1980, Miller et al (9) described hypoglycemia as a warning sign of bacterial sepsis, the mechanisms for which included depleted glycogen stores, impaired gluconeogenesis, and increased glucose utilization by the infecting pathogens. Based on the present study, infection workups must be performed when hypoglycemic patients are $>72.7$ years old, with a WBC of $>12,430 / \mu 1$ and a CRP of $>6.6 \mathrm{mg} / \mathrm{dl}$. Hypoglycemia with concurrent UTI is commonly observed in females (male:female $=0.44$ ), while patients with concurrent pneumonia have longer hospital stays than non-infected patients (21.7 vs. 12.7 days). The hypoglycemic patients with pneumonia exhibited higher WBC counts and CRP levels than concurrent UTI hypoglycemic patients in the current study (Table III).

There are various factors that contribute to hypoglycemia, including lack of recent food intake (44.6-51.8\%), alcohol consumption $(21.0 \%)$, the presence of coronary artery disease, concurrent infection, stroke, ARF, malignancy and recent hospital discharge $(2,8,10-12)$. However, hypoglycemia is not associated with the adverse outcome of acute myocardial infarction (6). Hypoglycemia with concurrent infection did not affect biochemistry levels (GOT, creatinine, sodium and potassium) when compared with hypoglycemic patients without concurrent infection. Furthermore, in concurrent UTI patients, missed meals exerted a reduced effect. 


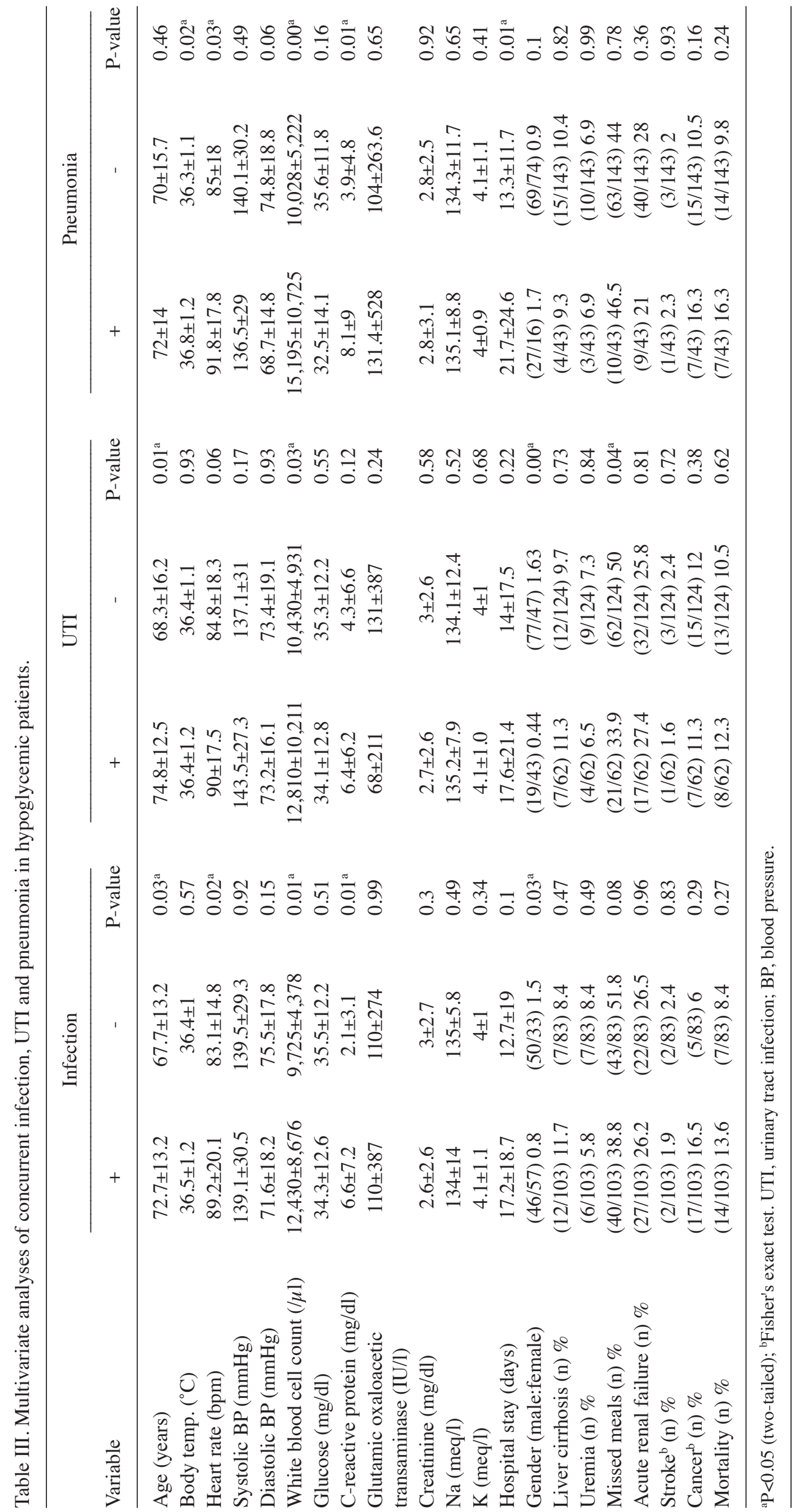


Impaired renal function and hepatic diseases are comorbidities that are associated with hypoglycemia $(1,3)$. In the current study, $62.4 \%$ of the hypoglycemic patients had impaired renal function and $26.3 \%$ of patients had ARF at the time of the hypoglycemic episode. Furthermore, concurrent infection did not increase the incidence of ARF in hypoglycemic patients. A German study in 2003 reported that $54.0 \%$ of the hypoglycemic patients exhibited renal impairment (13), while another study from southern Taiwan in 2006 reported that $5.7 \%$ of hypoglycemic patients had extensive liver disease (3). The current study demonstrated a higher incidence of liver cirrhosis in hypoglycemic patients with concurrent infection when compared with the non-infected hypoglycemic patients (11.7 vs. 8.4\%); however, the difference was not statistically significant. Hypo glycemic patients with liver cirrhosis had mortality rates 9.6 times higher than those without liver problems in the current study.

In the treatment of hypoglycemic patients, intravenous dextrose $(1 \mathrm{~g} / \mathrm{kg}$ body weight) is rapidly effective within a few min of administration. When a patient exhibits a serum glucose level of $<60 \mathrm{mg} / \mathrm{dl}$ and impaired mental state with decreased alertness, the administration of dextrose water is necessary. Thus, serial determination of the serum glucose level is mandatory.

Hypoglycemia is associated with various vascular diseases, such as coronary artery disease, stroke and peripheral arterial diseases (14). In the current study, $2.2 \%$ of hypoglycemic patients exhibited cerebrovascular accident at the time of the hypoglycemic episodes. In such a situation, intravenous dextrose fluid could not alleviate the neurologic deficit due to the initial hypoglycemia. In addition, elderly patients and those with preexisting co-morbidity conditions (chronic liver disease, end stage renal disease or adrenal insufficiency) are at risk for mortality $(6,15)$. Recurrent hypoglycemia commonly occurs in patients suffering from lack of recent food intake, coronary artery disease, concurrent infection or renal insufficiency. Hence it is proposed that they are admitted for observation and treatment (6). Although patients with hypoglycemia and a concurrent infection demonstrated a higher prevalence of cancer than non-infected patients ( 16.5 vs. $6.0 \%)$ in the current study, the difference was not statistically significant. Cancer, liver cirrhosis and concurrent stroke demonstrated greater impact on mortality in hypoglycemic patients when compared with concurrent infection in the present study (Fig. 1).

Although the length of hospital stay in the concurrent infection group was 4.5 days longer than that of the non-concurrent infection group, this difference was not statistically significant. However, concurrent infected status is not considered to be a precipitating factor resulting in poor outcomes. The overall mortality rate of hypoglycemic patients in the present study was $11.3 \%$.

There were certain limitations of the present study. First, it is a retrospective chart review during a brief time frame of 2 years and 3 months. Therefore, a longer period of study is required in future. In addition, with regard to the etiologies of hypoglycemia, it was difficult to define missed meals. Generally, cases were classified as resulting from missed meals by charted information (lack of regular intake, failure to feed themselves or failure by caregivers/families). Therefore, the missed meals data were gathered subjectively, rather than objectively. Furthermore, a multi-center study rather than a single center study is required to gather a credible number of studies.

In conclusion, hypoglycemic patients with concurrent infection were identified to be predominantly female and more elderly, with higher heart rates, WBC counts and CRP levels, typically exhibiting concurrent pneumonia. Cancer, liver cirrhosis and concurrent stroke were identified as more hazardous than concurrent infection in hypoglycemic patients. These characteristics must be considered in the initial management of hospitalized hypoglycemic patients. Furthermore, upon effective treatment of hypoglycemia and patients immediately regaining consciousness, it is proposed that hypoglycemic patients with concurrent infection, cancer, liver cirrhosis, or stroke require hospitalization and continued observation.

\section{References}

1. Ha WC, Oh SJ, Kim JH, Lee JM, Chang SA, Sohn TS and Son HS: Severe hypoglycemia is a serious complication and becoming an economic burden in diabetes. Diabetes Metab J 36: 280-284, 2012.

2. Lin YY, Hsu CW, Sheu WH, Chu SJ, Wu CP and Tsai SH: Risk factors for recurrent hypoglycemia in hospitalized diabetic patients admitted for severe hypoglycemia. Yonsei Med J 51: 367-374, 2010.

3. Su CC: Etiologies of acute hypoglycemia in a Taiwanese hospital emergency department. J Emerg Med 30: 259-261, 2006.

4. Van Biesen W, Vanholder R and Lameire N: Defining acute renal failure: RIFLE and beyond. Clin J Am Soc Nephrol 1: 1314-1319, 2006.

5. Lang $\mathrm{CH}$ and Dobrescu C: Sepsis-induced increases in glucose uptake by macrophage-rich tissues persist during hypoglycemia. Metabolism 40: 585-593, 1991.

6. Tsai SH, Lin YY, Hsu CW, Cheng CS and Chu DM: Hypoglycemia revisited in the acute care setting. Yonsei Med J 52: 898-908, 2011.

7. Leese GP, Wang J, Broomhall J, Kelly P, Marsden A, Morrison W, Frier BM and Morris AD; DARTS/MEMO Collaboration: Frequency of severe hypoglycemia requiring emergency treatment in type 1 and type 2 diabetes: A population-based study of health service resource use. Diabetes Care 26: 1176-1180, 2003.

8. Arinzon Z, Fidelman Z, Berner YN and Adunsky A: Infection-related hypoglycemia in institutionalized demented patients: A comparative study of diabetic and nondiabetic patients. Arch Gerontol Geriatr 45: 191-200, 2007.

9. Miller SI, Wallace RJ Jr, Musher DM, Septimus EJ, Kohl S and Baughn RE: Hypoglycemia as a manifestation of sepsis. Am J Med 68: 649-654, 1980.

10. Feher MD, Grout P, Kennedy A, Elkeles RS and Touquet R: Hypoglycemia in an inner-city accident and emergency department: A 12-month survey. Arch Emerg Med 6: 183-188, 1989.

11. Shorr RI, Ray WA, Daugherty JR and Griffin MR: Incidence and risk factors for serious hypoglycemia in older persons using insulin or sulfonylureas. Arch Intern Med 157: 1681-1686, 1997.

12. Waeschle RM, Moerer O, Hilgers R, Herrmann P, Neumann P and Quintel M: The impact of the severity of sepsis on the risk of hypoglycemia and glycemic variability. Crit Care 12: R129, 2008.

13. Holstein A, Plaschke A and Egberts EH: Clinical characterization of severe hypoglycemia-a prospective population-based study. Exp Clin Endocrinol Diabetes 111: 364-369, 2003.

14. Gitt AK, Bramlage P, Binz C, Krekler M, Plate T, Deeg E and Tschöpe D; DiaRegis Study Group: Hypoglycemia is more frequent in type 2 diabetic patients with co-morbid vascular disease: An analysis of the DiaRegis registry. Eur J Prev Cardiol 19: 765-772, 2012.

15. Parsaik AK, Carter RE, Myers LA, Basu A and Kudva YC: Hypoglycemia requiring ambulance services in patients with type 2 diabetes is associated with increased long term mortality. Endocr Pract 19: 29-35, 2013. 